\title{
Myc-binding protein orthologue interacts with AKAP240 in the central pair apparatus of the Chlamydomonas flagella
}

\author{
Venkatramanan G. Rao ${ }^{1}$, Ruhi B. Sarafdar', Twinkle S. Chowdhury ${ }^{1}$, Priyanka Sivadas², Pinfen Yang²,
} Prabhakar M. Dongre ${ }^{3}$ and Jacinta S. D'Souza ${ }^{1 *}$

\begin{abstract}
Background: Flagella and cilia are fine thread-like organelles protruding from cells that harbour them. The typical ' $9+2$ ' cilia confer motility on these cells. Although the mechanistic details of motility remain elusive, the dynein-driven motility is regulated by various kinases and phosphatases. A-kinase anchoring proteins (AKAPs) are scaffolds that bind to a variety of such proteins. Usually, they are known to possess a dedicated domain that in vitro interacts with the regulatory subunits (RI and RII) present in the CAMP-dependent protein kinase (PKA) holoenzyme. These subunits conventionally harbour contiguous stretches of a.a. residues that reveal the presence of the Dimerization Docking (D/D) domain, Catalytic interface domain and CAMP-Binding domain. The Chlamydomonas reinhardtii flagella harbour two AKAPs; viz., the radial spoke AKAP97 or RSP3 and the central pair AKAP240. Both these were identified on the basis of their RIl-binding property. Interestingly, AKAP97 binds in vivo to two RII-like proteins (RSP7 and RSP11) that contain only the D/D domain.
\end{abstract}

Results: We found a Chlamydomonas Flagellar Associated Protein (FAP174) orthologous to MYCBP-1, a protein that binds to organellar AKAPs and Myc onco-protein. An in silico analysis shows that the N-terminus of FAP174 is similar to those RIl domain-containing proteins that have binding affinities to AKAPs. Binding of FAP174 was tested with the AKAP97/RSP3 using in vitro pull down assays; however, this binding was rather poor with AKAP97/RSP3. Antibodies were generated against FAP174 and the cellular localization was studied using Western blotting and immunoflourescence in wild type and various flagella mutants. We show that FAP174 localises to the central pair of the axoneme. Using overlay assays we show that FAP174 binds AKAP240 previously identified in the C2 portion of the central pair apparatus.

Conclusion: It appears that the flagella of Chlamydomonas reinhardtii contain proteins that bind to AKAPs and except for the D/D domain, lack the conventional a.a. stretches of PKA regulatory subunits (RSP7 and RSP11). We add FAP174 to this growing list.

Keywords: Chlamydomonas reinhardtii, MYCBP-1, A-kinase anchoring proteins (AKAPs), Flagella, Central pair, FAP174

\section{Background}

Motile cilia/flagella propel eukaryotic cells in aqueous environment or circulate surrounding fluid. The movement is generated by a microtubule-based biological machine, the axoneme. Most axonemes adopt a ' $9+2$ ' format with 9 outer microtubule doublets encircling two

\footnotetext{
* Correspondence: jacinta@cbs.ac.in

'UM-DAE Centre for Excellence in Basic Sciences, Kalina campus, Santacruz

(E), Mumbai 400098, India

Full list of author information is available at the end of the article
}

microtubule singlets. The former associate with outer dynein arms (ODA), inner dynein arms (IDA) and radial spokes (RS), while the latter and a number of projections constitute the Central Pair (CP) apparatus. These structures along with other less evident complexes operate in concert to generate the rhythmic beating. And, cyclic adenosine monophosphate (cAMP) is a key 2nd messenger that regulates the movement. Studies with isolated axonemes of various mutants of the ODA, IDA, RS, CP and pharmacological inhibitors have implicated 
cAMP-dependent protein kinase (PKA) and other phosphoenzymes in the dynein-driven microtubule sliding [1-4]. Since isolated axonemes - without flagellar membrane and soluble contents - were used in the sliding assay, it was proposed that a network of phosphoenzymes anchored to the axoneme regulate dyneindriven motility [3-6].

PKA, a holoenzyme of two regulatory and two catalytic subunits, is anchored to scaffold protein; namely, the A-Kinase Anchoring Protein (AKAP). In 1982, AKAPs were first discovered as high affinity binding partners of the regulatory subunit of PKA [7]. Many more were discovered subsequently from several organisms and cell types by an overlay assay using the RII subunit [8]. In general, AKAPs are scaffold proteins sharing little sequence homology, but usually contain three common features - a region for targeting it to a particular micro-compartment, an Amphipathic helix (AH) that binds to a hydrophobic cleft of the Dimerization/ Docking domain (D/D) present on the PKA regulatory subunits, and additional motifs that recruit an array of molecules involved in signalling such as other protein kinases, phosphatases, phosphodiesterases, GSK3ß, and small GTPases [9]. This AH which binds to the D/D domain of the regulatory subunit of PKA and non-PKA proteins serves as a parameter for a protein to be designated as AKAP. Recently, an in silico approach was adopted to determine amphipathic helices containing proteins which could be candidate AKAPs [10].

Consistent with multiple implicated roles of PKA in ciliated cells, independent studies used RII overlays to reveal a number of AKAPs in this organelle, at least 7 AKAPs in the fibrous sheath surrounding the $9+2$ axoneme in mammalian sperms [11], one in cilia of the human respiratory tract [12] and two (AKAP97 and AKAP240) in the axoneme of Chlamydomonas flagella [13]. Analysis of flagellar mutants lacking specific axonemal complexes showed that AKAP97 is RSP3 in the RS complex, whereas AKAP240 resides in the CP. While this finding is consistent with the role of RS and the CP in regulating dynein motors, RSs isolated from Chlamydomonas flagella did not contain any PKA catalytic subunits [14]. Nonetheless, RSP3 and RS indeed harbour features related to PKA and AKAPs. The N-terminus of RSP3 anchors the RS to particular sites in the axoneme. Secondly, RSP3 forms a homodimer [15], each monomer containing an $\mathrm{AH}$ for interacting with RSP7 or RSP11 [16] that contains a RII domain but lack any features required for cAMP signalling or phosphorylation $[17,18]$. Therefore, the RS in Chlamydomonas flagella appears to utilize PKA anchoring mechanism to tether different molecular modules for the function of the RS.
Notably, a number of proteins with a RII domain have been discovered in mammalian cilia and flagella [18]. In addition, accumulated evidence indicates that RII harbours the $\mathrm{D} / \mathrm{D}$ domain. In fact, two conserved RS proteins contain what is known as the DPY30 domain that share a similar secondary and tertiary structure with the RII domain and bind amphipathic helices of AKAPs $[16,19,20]$. Another AKAP interactor, viz. Myc-binding protein-1 (MYCBP-1) was found to bind to the AH. MYC and MYCBP-1 complex acts as a transcriptional regulator, enhancing the transcription of genes controlled by the E-Box element and leading to erythrocyte differentiation [21, 22]. It was proposed that MYCBP-1, PKA and AKAP95 form a ternary complex in the nucleus negatively regulating the kinase activity [23]. MYCBP-1 operates outside the nucleus as well, especially during the interphase. It was shown that MYCBP1 interacts with a few AKAPs, such as AKAP149 in sperm mitochondria, its splice variant S-AKAP84 [24, 25], and BIG2, an AKAP in the trans-Golgi network [26]. Here, we show that FAP174 in Chlamydomonas flagella behaves like MYCBP-1 in associating with an AKAP, viz. AKAP240 in the C2 microtubule.

\section{Results}

FAP174 in Chlamydomonas is an MYCBP-1 homologue predicted to form a RII-like domain at the $\mathrm{N}$-terminus

Several studies have shown that MYCBP-1 is an AKAP interactor [24-27]. BLAST search with the human MYCBP-1 revealed a single homologue, FAP174 in the Chlamydomonas flagellar proteome and its presence in other non-ciliated organisms such as angiosperms. Phylogenetic analysis with representative MYCBP-1-like proteins from several organisms generated using MEGA6 [28] showed that FAP174 forms a cluster with the proteins from Volvox and protozoans (Fig. 1a). It also appears to have branched from the mammalian lineage of MYCBP-1. Sequence alignment using Clustal Omega [29] of FAP174 and MYCBP-1 from a few species of plants, animals, fungi and protozoans revealed substantial sequence identity or similarity (43-87\%) in the N-terminal region (Fig. 1b). This region also shares a.a. of similar properties with proteins containing the D/ D domain similar to that of RII and DPY-30 (Fig. 1c). Homology detection and secondary structure analysis revealed high sequence similarity with the $\mathrm{N}$-terminus that spanned the helix-turn-helix fold typical to the RII clan of proteins (Fig. 1c). This was done using HHpred [30]. The sequences with the RIIa D/D domain and DPY-30 domains that showed significant match were aligned using Multiple Sequence Alignment software (Clustal Omega), (Fig. 1c). From secondary structure prediction of FAP174, it appears that C-terminus is a helix with a strong propensity to form a coiled-coil, known for 


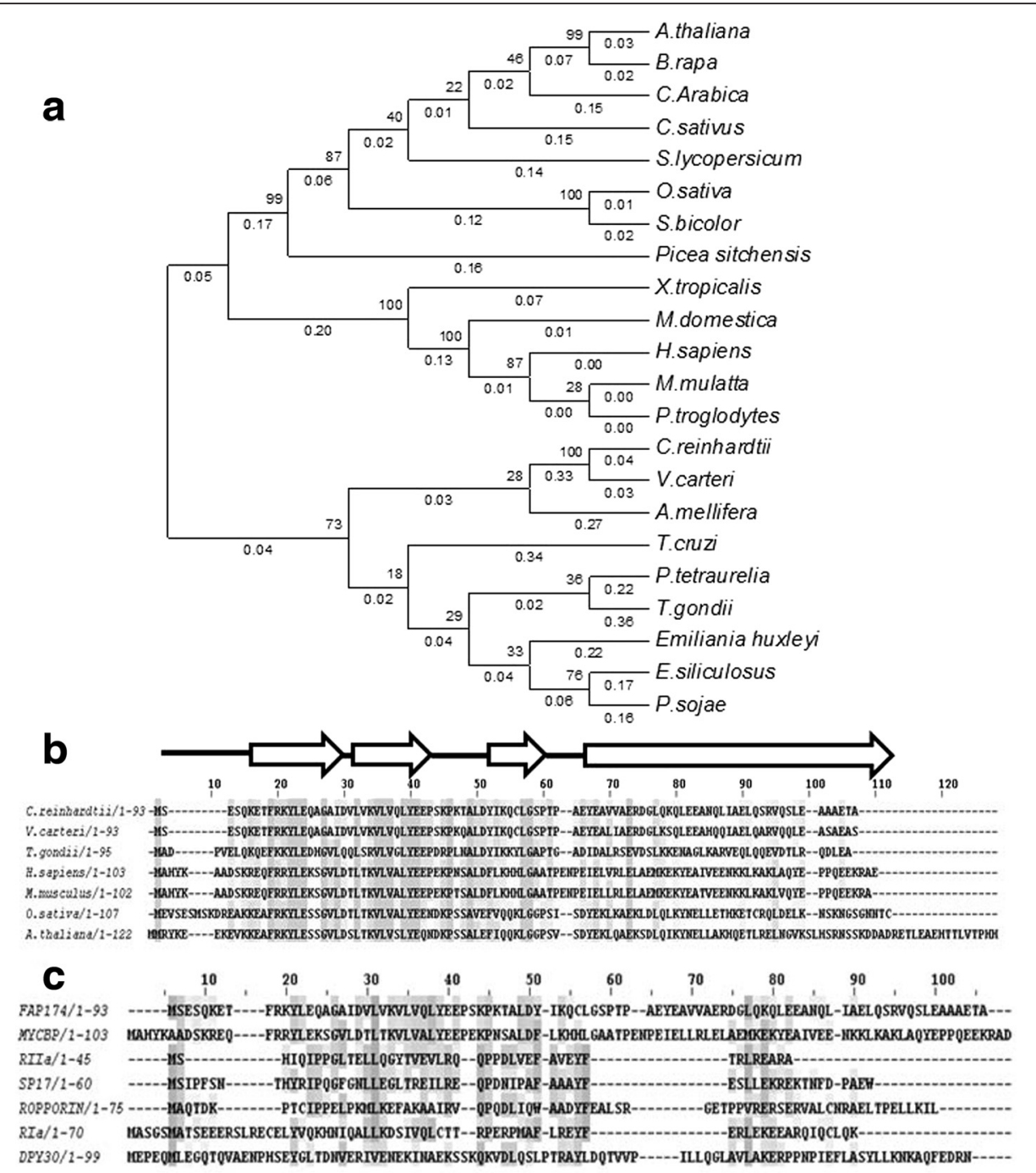

Fig. 1 FAP174 harbours a RII-like fold (a) A BLAST of FAP174 was carried out and representative MYCBP-1-like sequences from species of plants, animals, algae and protozoans were selected and a phylogenetic tree was generated using MEGA6. Organisms and their Accession numbers used for this exercise are C. reinhardtii ACR55627, V. carterii XP_002950671.1, B. rapa NP_001288931.1, A. thaliana NP_671849.1, C. arabica ADY38785.1, C. sativus XP_004172365.1, O. sativa EEC81635.1, S. bicolor XP_002459454.1, S. lycopersicon XP_004236796.1, P. sitchensis ABR18049.1, A. mellifera XP_624300.1, S. kowaleskii XP_002733639.1, X. tropicalis NP_001017035.1, M. Domestica XP_001364995.1, P. troglodytes XP_003949426.1, M. mulatta XP_001113156.2, H. sapiens BAA09338.1, P. tetraaurelia XP_001442915.1, T. cruzi XP_805155.1, P. sojae XP_009522570.1, A. anophagefferens XP_009033259.1, E. siliculosus CBN77061.1 and T. gondii XP_002370315.1. (b) Alignment of representative sequences from those used for the Phylogenetic analysis. The sequence homology is the highest at the N-terminal region that is predicted to fold into a helix-loop-helix structure as expected of dimerization and docking domains. The C-terminus of FAP174 shows a propensity to form coiled-coils. Identical residues are shaded, arrows represent the helix-forming residues and the lines represent coiled-coil forming residues. (c) Multiple alignment of FAP174 with proteins contianing the D/D domain using the best matches from HHpred. (http://toolkit.tuebingen.mpg.de/ hhpred). The sequences with the Rlla D/D domain and DPY-30 domains that showed significant match were aligned using Multiple Sequence Alignment software (Clustal Omega). Identical regions are shaded

protein-protein interaction. Therefore, we speculate that FAP174 has two molecular modules, one for binding an $\mathrm{AH}$ and one for partnering with proteins (Fig. 1b, c).

\section{Localization of FAP174}

The C. reinhardtii flagella contain two AKAPs; one localized to the RS and the other to the CP [13], (Fig. 2).
To determine whether FAP174 is interacting with the AKAP in the RS or CP, we over-expressed 6His-FAP174 in $E$. coli. The freshly purified recombinant protein migrated as $\sim 12 \mathrm{kDa}$ monomers in SDS-PAGE; after storage at $-20{ }^{\circ} \mathrm{C}$, it appeared as monomers and stable dimers. The purified protein was used to raise rabbit anti6 His-FAP174 polyclonal antibody. The affinity-purified 


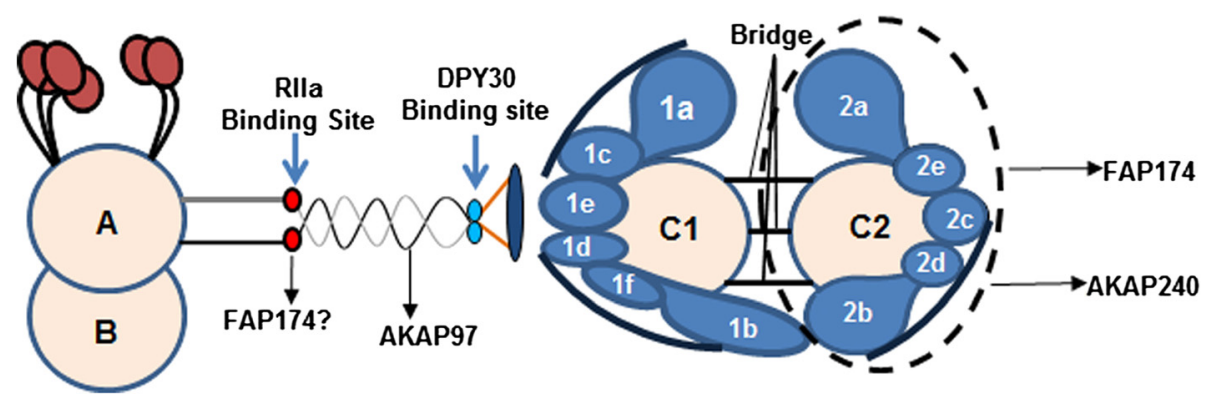

Fig. 2 The Radial spoke and Central pair AKAPs. Schematics depicting the two AKAPs in the $9+2$ axoneme. AKAP97 is RSP3 proposed to be a scaffold protein binding to radial spoke proteins (RSPS) with RII and DPY30 domains. The identity and partners of AKAP240 in C2 of the central pair apparatus remain elusive. This study attempts to identify these interactors. Only part of the $9+2$ axoneme cross section is illustrated. Also note, that the entire RSP harbours 23 different proteins; however, the figure depicts molecules significant for the current study

antibodies were used for probing flagella of WT and representative mutant strains lacking specific axonemal structures. Western blot analysis of axonemes showed that the antibody recognized a single $\sim 10 \mathrm{kDa}$ protein as expected of native FAP174 in WT axonemes (Fig. 3a,b). The band was present in pf14 lacking the RS and pf16 that is deficient in the $\mathrm{C} 1$ microtubule, but normal in the $\mathrm{C} 2$ of the CP apparatus. However, FAP174 is either absent or less abundant in pf15, pf18, pf19 and pf20 mutants that lacks the entire $\mathrm{CP}$ apparatus or is partially defective (Fig. 3a,b). This suggests that FAP174 is present in the C2 microtubule or the associated projections.

For immunofluorescence imaging, whole cells and the isolated Nucleo-Flagellar-Apparatus (NFA, intact flagella in conjunction with the nucleus and centriolar apparatus) were incubated with affinity-purified anti-6HisFAP174 antibody and mouse anti-acetylated tubulin antibody. As compared to the pre-immune serum control (Fig. 3c-e), both, anti-acetylated tubulin (using FITC filter) and anti-FAP174 (using CY3 filter) decorated the entire flagella, the basal bodies of WT cells and NFA (Fig. 3f-k). Notably, two pairs of spots are particularly prominent at the flagellar base. Consistent with the western blot, anti-6His-FAP174 did not decorate mutant pf18 flagella lacking the CP; but, still revealed the bright spots at the flagellar base (Fig. 3l-q). Taken together, these observations suggest the localization of FAP174 in the $\mathrm{C} 2$ part of the CP as AKAP240. The localization near the basal body and transition zone (TZ) could be the protein being transported to the flagella. In cells with less intense autofluorescence from plastids, FAP174 was also evident around the nucleus (Fig. 3r-t ) as reported previously for MYCBP-1 [21].

\section{In vitro interaction of FAP174 with the flagellar AKAPs}

We tested whether FAP174, like RII, can bind to flagellar AKAPs in overlay and in vitro 6His pull-down assays. The recombinant 6 His-FAP174 and GST-tagged RSP3 were co-expressed in E. coli BL21 (DE3) (arrowheads, Additional file 1: Figure S1). As a control, a clone coexpressing GST with FAP174 was generated. It was found that 6His-FAP174 pulled down GST-RSP3 and another polypeptide (though faint) of a similar molecular weight that was also seen in the GST tag control (Additional file 1: Figure S1). This suggested that the full-length GST-RSP3 did not interact with FAP174 under the current assay conditions. This led us to test the interaction of FAP174 with the RIIBinding $\mathrm{AH}$ in RSP3. We generated bacterial strains co-expressing 6His-FAP174 and the AH (spanning 96-180 a.a.) of RSP3, henceforth referred to as GSTRSP3 AH(96-180). Since low co-expression hindered the experiment, individual fusion proteins were purified (Fig. 4a). 6His-FAP174 pulled down the GST-RSP3 $\mathrm{AH}(96-180)$ with a low stoichiometry; but, not the GST tag (Fig. 4c). As a control, we tested the interaction between GST-RSP3 AH(96-180) and a non-relevant 6Histagged recombinant protein (i.e. Arl6) from Chlamydomonas reinhardtii (Fig. 4b). With no interaction observed for the negative control (Arl6), we found that FAP174 has rather weak affinity to the AH in RSP3.

It may be emphasized that an AKAP240 pull-down assay was not possible, since the AKAP240 gene and protein have not yet been identified. Therefore, we used an overlay assay to test the interaction of 6His-FAP174 and AKAP240 in the axonemes from WT, RS and CP mutants (pf14, pf15, pf18, pf19 and pf20). As compared with the only anti-His antibody control (Fig. 5a), RII overlays on axonemal blots revealed a band corresponding to AKAP240 in the WT, pf14 and partially defective pf20 CP mutant (Fig. 5b). In our gels, AKAP240 migrated near $260 \mathrm{kDa}$. This polypeptide was clearly absent in pf15, pf18 and pf19 axonemes that lack the entire CP apparatus. On the other hand, the band corresponding to RSP3 was seen in all the axonemes; except pf14. Like RII proteins, 6His-FAP174 in the overlay (Fig. 5c) binds 


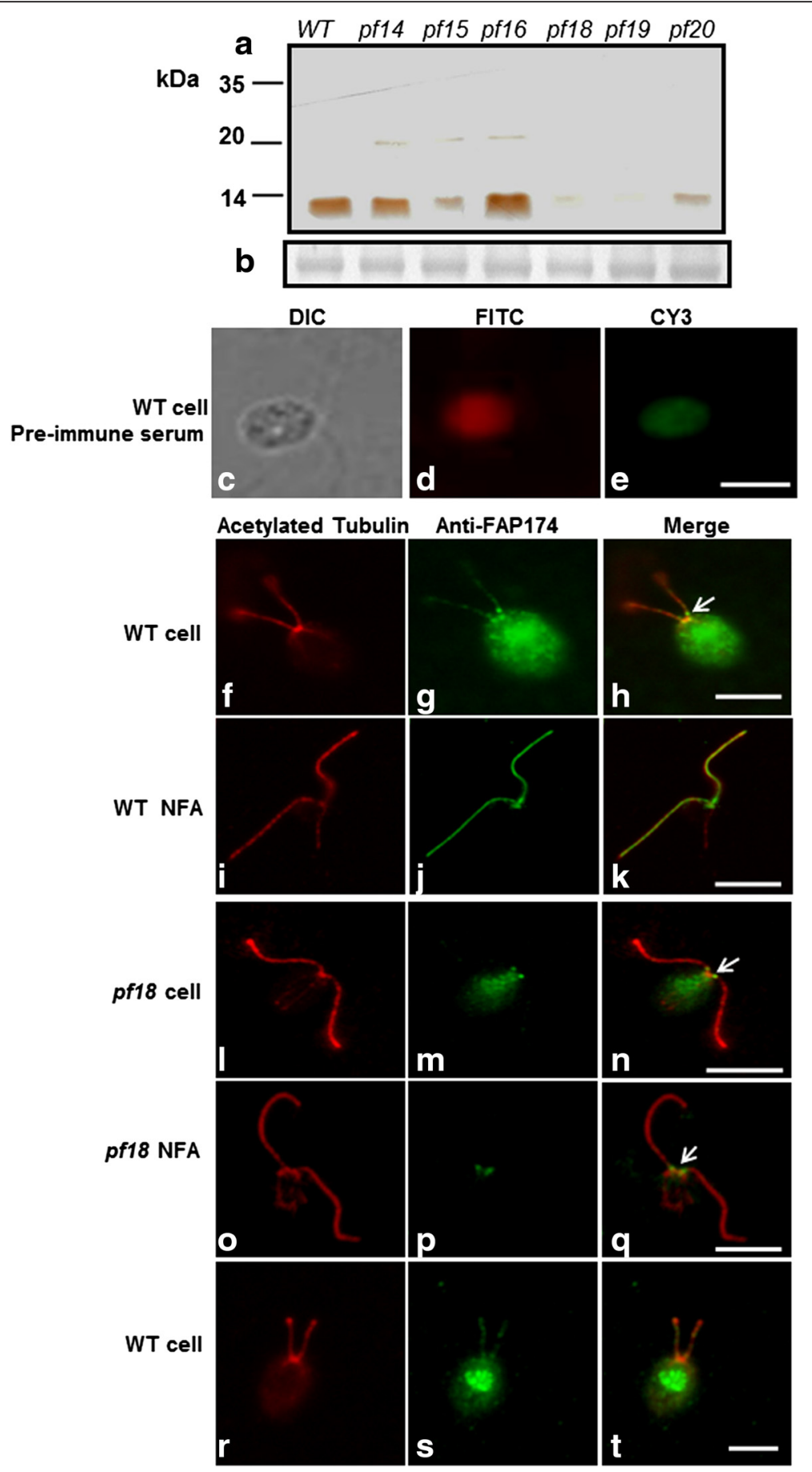

Fig. 3 Sub-flagellar localization of FAP174. (a) FAP174 Western blot of axonemes from WT and flagellar mutants. A polypeptide of $~ 10$ kDa was present in the axonemes of WT and the radial spokes mutant pf14; whereas, among the central pair mutants it was reduced in the pf15 and pf20, and absent in pf18 and pf19 that lack the central pair microtubules. The presence of FAP174 in pf16 axoneme that lack the C1 microtubule suggest that FAP174 associates with the C2 microtubule. (b) The Ponceau-stained tubulin bands show equal loading of axonemal proteins. (c-e) Immunofluorescent microscopy of whole cells using pre-immune serum as a control to show autofluorescence and non-specificity of the antibody (images captured at 63x magnification). (f-q) Immunofluorescent microscopy of FAP174 comparing whole cells and nucleo-flagella apparatus (NFA) from wild type and pf18. The samples were decorated with anti-acetylated tubulin or anti-FAP174 as indicated. FAP174 localizes to the flagella as well as to the base of flagella possibly the transition zone (arrows). FAP174 was not detectable in the pf18 flagella but appeared as the bright spot at the base of flagella. (r-t) Localization of FAP174 around nucleus is more prominent in cells with low autofluorescence background and one such representative image is depicted here. The scale bars in the figure indicates $10 \mu \mathrm{m}$ 


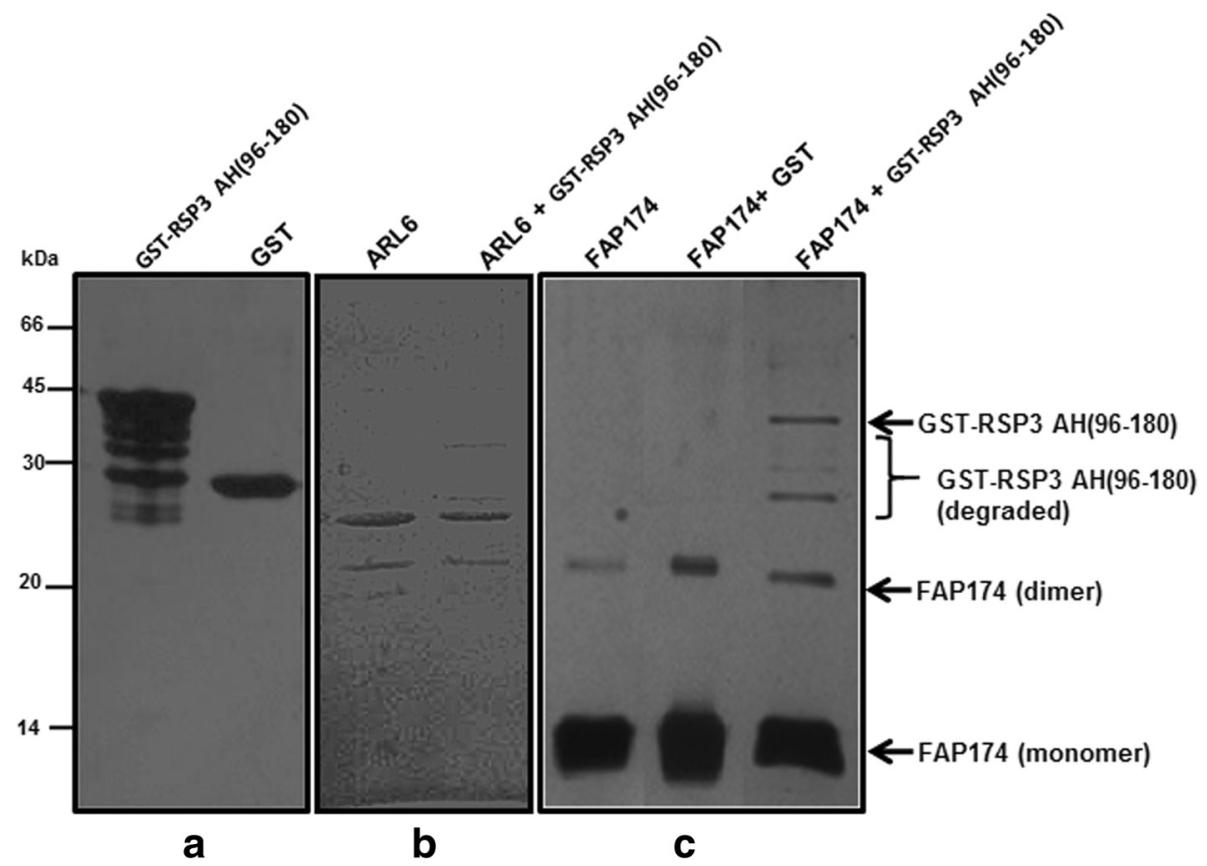

Fig. 4 Interaction of FAP174 with GST-RSP3-AH 96-180 in vitro. (a) Purified proteins used for the assay. (b) Negative control for the assay, which depicts no interaction between Arl6 and GST-RSP3 AH(96-180). (c) Co-purification of FAP174 with GST or GST-RSP3-AH 96-180. Testing the in vitro interaction of the GST-RSP3-AH 96-180 with 6His-FAP174 protein. Note the rather weak interaction as evidenced in the last lane (arrows)

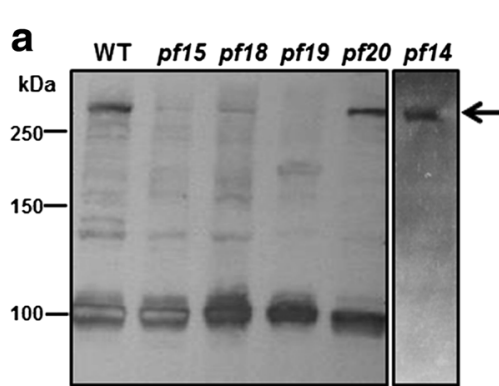

RII overlay

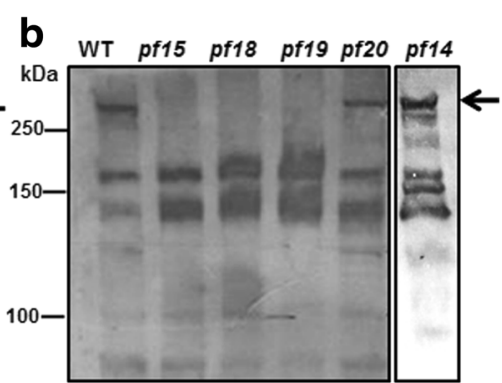

FAP174 overlay

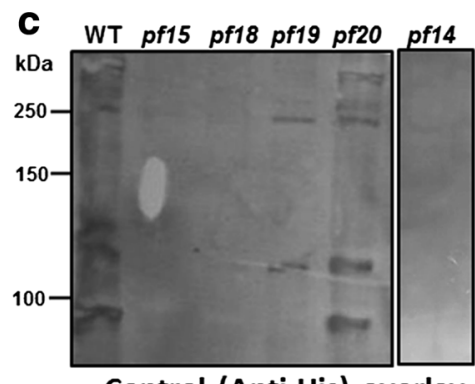

Control (Anti-His) overlay

Fig. 5 The recognition of AKAP240 by recombinant FAP174 and RII in vitro. (a) The blots were probed with recombinant 6His-tagged Rlla D/D protein and revealed by HRP-conjugated anti-His antibody. The polypeptide migrated above $250 \mathrm{kDa}$ in the axonemes of WT, pf14 and pf20 but were absent in mutants lacking the central pair, as expected of AKAP240. (b) 6His-FAP174 overlays also show the same band above $250 \mathrm{kDa}$. 6 His-FAP174 also reacted with two other proteins. The polypeptide migrated above $250 \mathrm{kDa}$ in the axonemes of WT, pf14 and pf20 but were absent in mutants lacking the central pair. (c) Control overlay blots with WT and mutant axonemes were probed with the anti-His antibody coupled to HRP without the interacting proteins 
to the expected $\sim 240 \mathrm{kDa}$ molecule in only WT and pf20 axonemes. Interestingly, though, unlike RIIa (D/D), 6 His-FAP174 did not evidently bind to RSP3 in the overlay, consistent with the low stoichiometry co-purification of FAP174 and RSP3 (Fig. 5b,c). The additional two unknown polypeptides of $\sim 150$ and $125 \mathrm{kDa}$ whose identity remains elusive were also detected in 6His-FAP174 overlay (Fig. 5c).

\section{Discussion}

MYCBP-1 was found to bind AKAPs in a few organelles, including the AKAPs in flagellar structures that are unique to sperms $[21,22,26]$. The current work demonstrates that the MYCBP-1 orthologue, FAP174, binds to AKAP240 in the axoneme of Chlamydomonas flagella. The findings expand the roles of RII-like domain in protein complex assembly and reveal new insight in the composition of the CP apparatus.

Independent lines of evidence indicate that FAP174 is a conserved structural component in a novel molecular complex in the $\mathrm{C} 2$ part of $\mathrm{CP}$ apparatus. It was first identified in Chlamydomonas flagella proteome project [31]. Western blots using the FAP174 specific antibodies showed that it is absent in mutant axonemes missing the C2 microtubule of the CP (Fig. 3a); but, is retained in the mutant axonemes missing the $\mathrm{C} 1$ fraction (Fig. 3a). While pf15 mutant which also shows reduced amounts of FAP174 is considered CP minus, its axoneme often contains an electron-dense core in the place of the $\mathrm{CP}$ [32]. It has been demonstrated recently that selective $\mathrm{CP}$ proteins remain in the core of CP mutants [33, 34]. The absence of RS, on the other hand, does not affect the assembly of FAP174 (Fig. 3a). Consistent with this, immunofluorescence microscopy show anti-FAP174 decorated the entire flagella of WT but not a $\mathrm{CP}$ mutant. Likewise, overlays showed that FAP174 and RIIa (D/D) recognize a $260 \mathrm{kDa}$ polypeptide in the axonemes of WT corresponding to AKAP240 present in the C2 microtubule and no band corresponding to the $97 \mathrm{kDa}$ RSP3 polypeptide. The latter result and its localization only to the central pair suggest that FAP174 is not a physiological interactor of RSP3.
MYCBP-1 was initially identified in the nucleus and the surrounding membranous networks for assisting cMYC trafficking between the compartments. It also forms a ternary complex with AKAPs and MYCBPAP in the nucleus. It seems that MYCBP-1 uses its RII-like domain to associate with AKAPs and its coiled-coil region to bind c-MYC and MYCBPAP. As far as the presence of FAP174 in basal body or TZ is concerned, the fluorescent signal could be indication of protein carried to these locations. Another interesting scenario could be there are additional AKAPs in the TZ or basal bodies anchoring FAP174 and are yet to be determined. Consistent with this notion, AKAP450 is present in the proteome of the human and fly centrosome $[35,36]$. In this regard, FAP174 is rather versatile as proteins that contain the D/D domain (RII clan) is involved in the assembly of several molecular complexes in distinct cellular compartments or organisms. To this extent, it would be very interesting to know the exact sub-flagellar localization of FAP174-AKAP240 complex and the molecular identity of AKAP240.

\section{Conclusions}

The current study has identified a MYCBP-1 like protein with D/D like domain localized in the central pair of the Chlamydomonas reinhardtii flagella. Similar to its mammalian equivalent, the $\mathrm{D} / \mathrm{D}$ domain facilitates its binding to AKAPs. The study further adds FAP174 as a new protein to the $\mathrm{C} 2$ microtubule protein complexes. Our further efforts are to characterize this AKAP-FAP174 complex, find the molecular identity of AKAP240 and explore the role of this complex in the motility of the $9+2$ cilia.

\section{Methods}

The biochemical reagents and media components were obtained from Amresco (USA), SRL (India) and Merck Millipore (India). Ni-NTA Agarose used for protein purification was obtained from Qiagen (Germany). The primers for PCR were obtained from Merck Millipore (Genei, India). Also see Table 1 for list of clones used in the current study.

Table 1 List of genes and proteins used/generated in the current study

\begin{tabular}{llllll}
\hline No. & Insert & Vector & Protein product & Comment & Ref \\
\hline 1 & fap174 & pET28a & 6His FAP174 & c-Myc Binding protein ortholog & Current study \\
2 & RSP3 & pGEX2T & GST-RSP3 & Radial spoke AKAP full length & {$[17]$} \\
3 & Arsp3 (96-180) & pGEX2T & GST-RSP3-AH 96-180 & $\begin{array}{l}\text { Truncated RSP-3 with Amphipathic helix (96-180 a.a.) } \\
\text { that binds to proteins with Dimerization and Docking } \\
\text { domains }\end{array}$ & {$[14]$} \\
4 & arl6 & pET28a & ARL6 & Full length GTP-binding protein & From Jacinta D'Souza laboratory \\
5 & rlla D/D & pET15b & Rlla D/D & $\begin{array}{l}\text { Regulatory subunit of PKA, truncated protein (1-44 a.a.) } \\
\text { with Dimerization and Docking domain }\end{array}$ & Gift from Susan Taylor laboratory [39] \\
\hline
\end{tabular}




\section{Chlamydomonas reinhardtii cultures}

The wild type (cc124) and mutant strains deficient in the RS (pf14) and CP (pf14, pf15, pf16, pf18, pf19, and pf20) and cc620, cc621 were procured from Chlamydomonas Resource Center (University of Minnesota). All cells were grown in liquid TAP medium with continuous light under shaking conditions at $25^{\circ} \mathrm{C}$ [37].

\section{Cloning of fap174 gene, induction, over-expression and purification of FAP174 and other proteins}

Primers were designed specific to fap174 gene with NdeI and EcoRI as flanking restriction sites (FAP174 Nde FP: CATATGATGTCGGAGTCG and FAP174 Eco RP: GAATTCTTATGCAGTCTCCGC). Using the C. reinhardtii S1D2 cDNA library as template in a PCR reaction, the amplicons so obtained were cloned into the Nde1 and EcoR1 sites of the pET28a vector and the resultant construct transformed into competent E. coli BL21(DE3) cells (GenBank Accession no. FJ986299). The 6His-tagged FAP174 recombinant protein was induced using IPTG. The protein was purified using $\mathrm{Ni}$ NTA Chromatography with minor modifications to the protocol [38]. Chlamydomonas RSP-3 and the nucleotide region containing RII-binding AH spanning 96-180 a.a. were cloned in pGEX-2 T vector. Both fusion proteins were induced using $1 \mathrm{mM}$ IPTG and purified using Glutathione-Sepharose 4B (GE Healthcare). Rat RIIa $\mathrm{D} / \mathrm{D}$ (coding for 1-44 a.a.) cloned in $\mathrm{pET} 15 \mathrm{~b}$ vector was a kind gift of Dr. Susan Taylor (UCSD, USA). The protein was purified according to procedures described previously [39].

\section{Extraction of flagella, axonemes and nucleo-flagella apparatus}

Chlamydomonas cells were grown till mid-log phase and harvested by centrifugation (1,100 $\mathrm{g} / 5 \mathrm{mins})$. The flagella were extracted according to standard protocol [40]. For axoneme extraction, the flagella were de-membranated using $0.5 \%$ IGEPAL CA0630 (Sigma) in HMDEK buffer followed by centrifugation $(16,000 \mathrm{~g} / 20 \mathrm{mins}$.). For preparing the Nucleo-Flagella-Apparatus (NFA), [41], autolysin-treated cells were loaded on glass slides and treated with $1 \mathrm{X}$ Microtubule stabilising buffer (MT, $30 \mathrm{mM}$ Tris-Cl, $5 \mathrm{mM} \mathrm{MgSO}$, $5 \mathrm{mM}$ EGTA, $25 \mathrm{mM}$ $\mathrm{KC} 1,1 \mathrm{mM}$ dithiothreitol, $\mathrm{pH}$ 7.3) containing 1 \% IGEPAL CA0630. Later, the sample on the coverslips was fixed with 1X MT buffer containing $2 \%$ paraformaldehyde followed by washing once with 1X MT and immersing in methanol at $-20^{\circ} \mathrm{C}$ for $5 \mathrm{~min}$. Later, the NFA's were used in the indirect immunofluorescence studies.

\section{Antibody production and indirect immunofluorescence} Purified 6His-tagged FAP174 protein was used as an antigen to immunize rabbits. The antibody generation and affinity purification of anti-FAP174 antibodies was done as a service by Merck-Millipore (Genei, India). These affinity-purified antibodies were used for immunofluorescence assays. For indirect immunofluorescence assay, cc124 cell suspensions were applied to polyethylenimine (PEI)-treated coverslips. Cells were allowed to adhere and subsequently washed to remove those that have not adhered. The coverslip was immersed in methanol at $-20{ }^{\circ} \mathrm{C}$ for $5 \mathrm{~min}$. The airdried coverslips were washed and treated with blocking solution (3 \% BSA in Phosphate-Buffered Saline, PBS) and then rabbit anti-6His-FAP174 antibody (1:1500 dilution) and mouse anti-acetylated alpha tubulin antibody (1:500 dilution Abcam, 6-11B-1 clone) were added at the same time for $1 \mathrm{~h}$ followed by washing with PBST (PBS + $0.05 \%$ Tween-20). Then, incubation with antimouse Alexa flour-568 and anti-rabbit Alexa flour488 (1:100) (Molecular probes, USA) was carried out for $1 \mathrm{~h}$. After washes, coverslips were mounted with a drop of Prolonged Gold Anti-fade reagent (Molecular Probes, USA) on glass slides and observed using Nikon 90i microscope using plan Flour $63 x / 1.25$ oil immersion objective and appropriate filters. When the primary antibody was replaced with a pre-immune antibody and the entire procedure repeated as above, these cells served as a control.

\section{ECL and overlay assays}

Purified protein and flagellar/axonemal extracts were electrophoresed on SDS-PAGE gels and transferred onto a nitrocellulose membrane. The membrane was stained for the presence of proteins with Ponceau $S$ and subsequently blocked with $3 \%$ BSA in TBS $+0.05 \%$ Tween20 (TBST). For overlay assay, the membrane was overlaid with $\sim 20 \mu \mathrm{g} / \mathrm{ml}$ purified FAP174 or RIIa (D/D) protein after blocking. The control blot (anti-His only) was not overlaid with any protein with the subsequent steps same as that of the test blot. Subsequent steps in both the cases involved washing 3 times with $1 \%$ BSA in TBST and probing with primary and secondary antibodies at appropriate dilutions. The membranes were developed using ECL-Advance (GE Healthcare, USA) and the signal was revealed using conventional X-Ray plates, developers and fixers.

\section{Protein-protein interaction assays (Co-expression, His-Pull-down assay)}

For co-expression and pull-down studies, modified procedure of Yang and Yang (2006) was followed. Briefly, plasmids harbouring different genes [fap174, rsp3 and $\Delta r s p 3(96-180)]$ and selection antibiotics were cotransformed into E. coli BL21(DE3) cells and the proteins over-expressed after induction with $1 \mathrm{mM}$ IPTG. As a control, empty pGEX2T plasmid was co-transformed 
with the fap174 construct. Upon appropriate selection, positive clones were used to pull-down the 6His-FAP174 protein with Ni-NTA resin. Pull-down assays were carried out using respective lysates from co-transformants or pure proteins (FAP174, RSP3 or GST-RSP3-AH 96-180) or GST as a control). For His-pull-down assays, the cell lysates or purified proteins were incubated with the Ni-NTA resin at $4{ }^{\circ} \mathrm{C}$ for $1 \mathrm{~h}$, followed by three washes with assay buffer $(25 \mathrm{mM}$ Tris-Cl, $\mathrm{pH} 7.5$, $150 \mathrm{mM} \mathrm{NaCl}, 5$ \% Sucrose, $0.1 \%$ Triton-X, $0.1 \mathrm{mM}$ PMSF, $50 \mathrm{mM}$ Imidazole). The interactor was then added and the mixture incubated at $4{ }^{\circ} \mathrm{C}$ for $2 \mathrm{~h}$, followed by three washes with the same buffer. Subsequent electrophoresis and silver staining revealed the interactors.

\section{Additional file}

Additional file 1: Figure S1. Testing the interaction of FAP174 with GST-tagged RSP3 in vitro. (A) Co-expression of FAP174 with GST or GSTtagged RSP3. The first, second and fourth lanes are the crude lysates with independently expressing FAP174, GST and GST-RSP3 proteins (see arrowheads); the third lane is the clone over-expressing both the FAP174 and GST proteins; while, the last lane is the lysate from the clone over-expressing both FAP174 and GST-RSP3 (see arrowheads). (B) FAP174 (arrow at the bottom of the gel) was co-purified with GST-RSP3 (arrow at the top of the gel) but not with GST alone. FT, $W$ and $E$ are Flow-through, Wash and Elutes, respectively. Note that there no interaction between full-length RSP3 and FAP174. (PNG 379 kb)

\section{Abbreviations}

AH, amphipathic helix; AKAP, A-Kinase anchoring protein; ARL6, ADP ribosylation factor-like 6 protein; BIG2, brefeldin a-inhibited guanine nucleotideexchange factor 2; BLAST, basic local alignment search tool; BSA, bovine serum albumin; CAMP, cyclic adenosine monophosphate; c-Myc, myelocytomatosis; CP, central pair; D/D domain, dimerization docking domain; DPY-30, Dumpy-30; ECL- Enhanced chemiluminesence; FAP174, flagella associated protein 174; GSK3ß, glycogen synthase kinase 3beta; GST, glutathione-Stransferase; IDA, inner dynein arms; IPTG, isopropyl $\beta$-D-1-thiogalactopyranoside; kDa, kilo Dalton; MEGA6, molecular evolutionary genetics analysis version6; MT buffer, microtubule stabilising buffer; MYCBP-1, MYCBinding protein-1; MYCBPAP, Myc-binding protein associated protein; NFA, nucleoflagellar apparatus; Ni-NTA, nickel-nitrilotriacetic acid; ODA, outer dynein arms; PBS, phosphate buffered saline; $P C R$, polymerase chain reaction; PEI, polyethyleneimine; PKA, CAMP-dependent protein kinase; PMSF, phenylmethylsulphonyl fluoride; RS, radial spoke; RSP, radial spoke protein; SDS-PAGE, sodium dodecyl sulphate-polyacrylamide gel electrophoresis; TBS, Tris buffered saline; TZ, transition zone; WT, Wild type

\section{Acknowledgement}

The authors VGR and JSD wish to thank Anne Gaillard (San Houston State Univ., USA) for the advice in reproducing the RII overlays; Dennis Diener (Yale Univ., USA) for providing the RSP3 expression construct; Prof. Susan Taylor (UCSD, USA) for providing the rat Rlla(D/D) construct.

\section{Funding}

VGR and JSD wish to thank the Department of Atomic Energy, India for funding this work. JSD wishes to thank the Department of Biotechnology, India for partial funding of this work (Grant No. BT/PR3159/BRB/10/960/2011).

\section{Availability of data and materials}

The nucleotide sequence of fap174 was submitted to NCBI's GenBank database with the Accession number FJ986299. The E. coli overexpression vector (pET28a) in which fap174 gene was cloned would be available upon request.

\section{Authors' contribution}

VGR performed the molecular, biochemical studies and immunoassays. RBS, TSC, PS, PMD participated in the experiments and PY participated in designing of experiments. JSDS conceived, planned, coordinated and drafted the manuscript. All the authors have read the final version of the manuscript.

\section{Competing interests}

None of the authors have any competing interests to declare.

\section{Consent for publication}

"Not applicable"

\section{Ethics approval and consent to participate}

"Not applicable"

\section{Author details}

'UM-DAE Centre for Excellence in Basic Sciences, Kalina campus, Santacruz (E), Mumbai 400098, India. ${ }^{2}$ Wehr Life Sciences, Marquette University, P.O. Box 1881, Milwaukee, WI 53201-1881, USA. ${ }^{3}$ Department of Biophysics, University of Mumbai, Kalina campus, Santacruz (E), Mumbai 400098, India.

Received: 25 November 2015 Accepted: 2 June 2016

Published online: 10 June 2016

\section{References}

1. Howard DR, Habermacher G, Glass DB, Smith EF, Sale WS. Regulation of Chlamydomonas flagellar dynein by an axonemal protein kinase. J Cell Biol. 1994;127(6 Pt 1):1683-92

2. Habermacher G, Sale WS. Regulation of flagellar dynein by an axonemal type-1 phosphatase in Chlamydomonas. J Cell Sci. 1996;109(Pt 7):1899-907.

3. Smith EF. Regulation of flagellar dynein by calcium and a role for an axonemalcalmodulin and calmodulin-dependent kinase. Mol Biol Cell. 2002;13(9):3303-13

4. Gokhale A, Wirschell M, Sale WS. Regulation of dynein-driven microtubule sliding by the axonemal protein kinase CK1 in Chlamydomonas flagella. J Cell Biol. 2009;186(6):817-24.

5. Porter ME, Sale WS. The $9+2$ axoneme anchors multiple inner arm dyneins and a network of kinases and phosphatases that control motility. J Cell Biol. 2000;151(5):F37-42.

6. Mitchell BF, Pedersen LB, Feely M, Rosenbaum JL, Mitchell DR. ATP production in Chlamydomonas reinhardtii flagella by glycolytic enzymes. Mol Biol Cell. 2005;16(10):4509-18.

7. Theurkauf WE, Vallee RB. Molecular characterization of the cAMP-dependent protein kinase bound to microtubule-associated protein 2. J Biol Chem. 1982;257(6):3284-90.

8. Lohmann SM, DeCamilli P, Einig I, Walter U. High-affinity binding of the regulatory subunit (RII) of CAMP-dependent protein kinase to microtubuleassociated and other cellular proteins. Proc Natl Acad Sci U S A. 1984;81(21):6723-7.

9. Scott JD, Dessauer CW, Tasken K. Creating order from chaos: cellular regulation by kinase anchoring. Annu Rev Pharmacol Toxicol. 2013;53:187-210.

10. Burgers PP, van der Heyden MA, Kok B, Heck AJ, Scholten A. A systematic evaluation of protein kinase A-A-kinase anchoring protein interaction motifs. Biochemistry. 2015;54(1):11-21.

11. Carnegie GK, Means CK, Scott JD. A-kinase anchoring proteins: from protein complexes to physiology and disease. IUBMB Life. 2009;61(4):394-406.

12. Kultgen PL, Byrd SK, Ostrowski LE, Milgram SL. Characterization of an A-kinase anchoring protein in human ciliary axonemes. Mol Biol Cell. 2002;13(12):4156-66.

13. Gaillard AR, Diener DR, Rosenbaum JL, Sale WS. Flagellar radial spoke protein 3 is an A-kinase anchoring protein (AKAP). J Cell Biol. 2001;153(2):443-8.

14. Yang P, Diener DR, Yang C, Kohno T, Pazour GJ, Dienes JM, Agrin NS, King SM, Sale WS, Kamiya, R et al. Radial spoke proteins of Chlamydomonas flagella. J Cell Sci. 2006;119(Pt 6):1165-74.

15. Wirschell M, Zhao F, Yang C, Yang P, Diener D, Gaillard A, Rosenbaum JL, Sale WS. Building a radial spoke: flagellar radial spoke protein 3 (RSP3) is a dimer. Cell Motil Cytoskeleton. 2008;65(3):238-48.

16. Sivadas P, Dienes JM, St Maurice M, Meek WD, Yang P. A flagellar A-kinase anchoring protein with two amphipathic helices forms a structural scaffold in the radial spoke complex. J Cell Biol. 2012;199(4):639-51. 
17. Yang C, Yang P. The flagellar motility of Chlamydomonas pf25 mutant lacking an AKAP-binding protein is overtly sensitive to medium conditions. Mol Biol Cell. 2006;17(1):227-38.

18. Newell AE, Fiedler SE, Ruan JM, Pan J, Wang PJ, Deininger J, et al. Protein kinase A RII-like (R2D2) proteins exhibit differential localization and AKAP interaction. Cell Motil Cytoskeleton. 2008;65(7):539-52.

19. Chen Y, Wan B, Wang KC, Cao F, Yang Y, Protacio A, et al. Crystal structure of the $\mathrm{N}$-terminal region of human Ash2L shows a winged-helix motif involved in DNA binding. EMBO Rep. 2011;12(8):797-803.

20. Gopal R, Foster KW, Yang P. The DPY-30 domain and its flanking sequence mediate the assembly and modulation of flagellar radial spoke complexes. Mol Cell Biol. 2012;32(19):4012-24.

21. Taira T, Maeda J, Onishi T, Kitaura H, Yoshida S, Kato H, et al. AMY-1, a novel C-MYC binding protein that stimulates transcription activity of C-MYC. Genes Cells. 1998:3(8):549-65.

22. Furusawa M, Onishi T, Taira T, Iguchi-Ariga SM, Ariga H. AMY-1 is a trigger for the erythrocyte differentiation of K562 cells. Int J Oncol. 2000;16(2):339-45

23. Furusawa M, Taira T, Iguchi-Ariga SM, Ariga H. AMY-1 interacts with SAKAP84 and AKAP95 in the cytoplasm and the nucleus, respectively, and inhibits CAMP-dependent protein kinase activity by preventing binding of its catalytic subunit to A-kinase-anchoring protein (AKAP) complex. J Biol Chem. 2002;277(52):50885-92.

24. Furusawa M, Ohnishi T, Taira T, Iguchi-Ariga SM, Ariga H. AMY-1, a c-Mycbinding protein, is localized in the mitochondria of sperm by association with S-AKAP84, an anchor protein of cAMP-dependent protein kinase. J Biol Chem. 2001;276(39):36647-51.

25. Yukitake H, Furusawa M, Taira T, Iguchi-Ariga SM, Ariga H. AAT-1, a nove testis-specific AMY-1-binding protein, forms a quaternary complex with AMY-1, A-kinase anchor protein 84, and a regulatory subunit of CAMPdependent protein kinase and is phosphorylated by its kinase. J Biol Chem. 2002;277(47):45480-92

26. Ishizaki R, Shin HW, Iguchi-Ariga SM, Ariga H, Nakayama K. AMY-1 (associate of Myc-1) localization to the trans-Golgi network through interacting with BIG2, a guanine-nucleotide exchange factor for ADP-ribosylation factors. Genes Cells. 2006:11(8):949-59.

27. Yukitake H, Furusawa M, Taira T, Iguchi-Ariga SM, Ariga H. AMAP-1, a novel testis-specific AMY-1-binding protein, is differentially expressed during the course of spermatogenesis. Biochim Biophys Acta. 2002;1577(1):126-32.

28. Tamura K, Stecher G, Peterson D, Filipski A, Kumar S. MEGA6: Molecular Evolutionary Genetics Analysis version 6.0. Mol Biol Evol. 2013;30(12):2725-9.

29. Sievers F, Wilm A, Dineen D, Gibson TJ, Karplus K, Li W, et al. Fast, scalable generation of high-quality protein multiple sequence alignments using Clustal Omega. Mol Syst Biol. 2011;7:539.

30. Meier A, Soding J. Automatic Prediction of Protein 3D Structures by Probabilistic Multi-template Homology Modeling. PLoS Comput Biol. 2015;11(10):e1004343.

31. Pazour GJ, Agrin N, Leszyk J, Witman GB. Proteomic analysis of a eukaryotic cilium. J Cell Biol. 2005:170(1):103-13.

32. Mitchell DR. The flagellar central pair apparatus. In: Cell Motility and Behaviour, Witman GB, editors. The Chlamydomonas Source book, vol. 3 Secondth ed. Academic; 2009. p. 235-52.

33. Adams GM, Huang B, Piperno G, Luck DJ. Central-pair microtubular complex of Chlamydomonas flagella: polypeptide composition as revealed by analysis of mutants. J Cell Biol. 1981;91(1):69-76.

34. Lechtreck KF, Gould TJ, Witman GB. Flagellar central pair assembly in Chlamydomonas reinhardtii. Cilia. 2013;2(1):15.

35. Alves-Cruzeiro JM, Nogales-Cadenas R, Pascual-Montano AD. CentrosomeDB: a new generation of the centrosomal proteins database for Human and Drosophila melanogaster. Nucleic Acids Res. 2014;42(Database issue):D430-6.

36. Nogales-Cadenas R, Abascal F, Diez-Perez J, Carazo JM, Pascual-Montano A. CentrosomeDB: a human centrosomal proteins database. Nucleic Acids Res. 2009;37(Database issue):D175-80

37. Khona DK, Rao VG, Motiwalla MJ, Varma PC, Kashyap AR, Das K, et al. Anomalies in the motion dynamics of long-flagella mutants of Chlamydomonas reinhardtii. J Biol Phys. 2013;39(1):1-14.

38. Sengupta K, Kamdar RP, D'Souza JS, Mustafi SM, Rao BJ. GTP-induced conformational changes in translin: a comparison between human and Drosophila proteins. Biochemistry. 2006;45(3):861-70.
39. Kinderman FS, Kim C, von Daake S, Ma Y, Pham BQ, Spraggon G, et al. A dynamic mechanism for AKAP binding to RII isoforms of CAMP-dependent protein kinase. Mol Cell. 2006;24(3):397-408.

40. Witman GB. Isolation of Chlamydomonas flagella and flagellar axonemes. Methods Enzymol. 1986;134:280-90.

41. Wright RL, Salisbury J, Jarvik JW. A nucleus-basal body connector in Chlamydomonas reinhardtii that may function in basal body localization or segregation. J Cell Biol. 1985;101(5 Pt 1):1903-12.

\section{Submit your next manuscript to BioMed Central and we will help you at every step:}

- We accept pre-submission inquiries

- Our selector tool helps you to find the most relevant journal

- We provide round the clock customer support

- Convenient online submission

- Thorough peer review

- Inclusion in PubMed and all major indexing services

- Maximum visibility for your research

Submit your manuscript at www.biomedcentral.com/submit
) Biomed Central 